\title{
Cloud Computing and Dynamic Resource Allocation for Multimedia Applications
}

\author{
Yifeng He, ${ }^{1}$ Ling Guan, ${ }^{1}$ Wenwu Zhu, ${ }^{2}$ and Ivan Lee $^{3}$ \\ ${ }^{1}$ Department of Electrical and Computer Engineering, Ryerson University, Toronto, ON, Canada M5B 2K3 \\ ${ }^{2}$ Department of Computer Science, Tsinghua University, Beijing 100084, China \\ ${ }^{3}$ School of Computer and Information Science, University of South Australia, Adelaide, SA 5095, Australia
}

Correspondence should be addressed to Yifeng He, yhe@ee.ryerson.ca

Received 22 December 2011; Accepted 22 December 2011

Copyright (c) 2012 Yifeng He et al. This is an open access article distributed under the Creative Commons Attribution License, which permits unrestricted use, distribution, and reproduction in any medium, provided the original work is properly cited.

We have witnessed significant advances in multimedia applications due to the rapid increase in digital media, computing power, communication speed, and storage capacity. Multimedia has become an indispensable aspect in contemporary daily life. Recently, cloud computing technology has offered great opportunities for multimedia applications. The performance of the multimedia applications can be significantly improved by optimizing the various resources in the multimedia system. The papers selected for this special issue represent a good panel for addressing the resource allocation problems in multimedia applications. We would like to thank the authors for their excellent contributions. We are grateful to all reviewers for their constructive comments which help to improve the quality of the papers.

This special issue contains five papers, in which two papers are related to cloud-based multimedia applications, two papers deal with Peer-to-Peer (P2P) video streaming systems, and one paper addresses the resource allocation problem in wireless networks.

In the paper entitled "Multi-objective genetic algorithm for task assignment on heterogeneous nodes," C. B. P. Del Notario et al. present a task assignment strategy based on genetic algorithms for a client-cloud multimedia system. Specifically, the task execution quality is maximized under the constraints of energy and bandwidth. In the video processing scenario, the proposed method employs trans-coding to provide a tradeoff between video quality and processing and bandwidth demands.

In the paper entitled "Towards an automatic parametertuning framework for cost optimization on video encoding cloud," X. Li et al. conduct an empirical study on video encoding cloud, targeting an optimization framework to minimize H.264 encoding cost by tuning the key parameters. The experiment results show that the tested parameters can be independently tuned to minimize the encoding cost, which makes the automatic parameter-tuning framework feasible and promising for video encoding cloud.

In the paper entitled "Improving streaming capacity in multi-channel P2P VoD systems via intra-channel and crosschannel resource allocation," Y. He and L. Guan. present novel methods to improve the streaming capacity for a multichannel P2P video-on-demand (VoD) system by efficiently utilizing both intrachannel and cross-channel resources. The intrachannel resource allocation problem can be formulated into a linear programming (LP) problem. The cross-channel resource allocation can be enabled by both optimal server upload allocation among channels and cross-channel sharing of peer upload bandwidths.

In the paper entitled "Performance evaluation of an object management policy approach for P2P networks," D. Vieira et al. present an object management approach for video web cache in a P2P network, taking advantage of object's metadata, for example, video popularity, and object's encoding techniques, for example, scalable video coding (SVC). In addition, the paper examines the impact of churn on the performance of object management policies.

In the paper entitled "A gain-computation enhancements resource allocation for heterogeneous service flows in IEEE 802.16 m mobile networks," W. B. Hassen and M. Afif. examine resource allocation problems for orthogonal-frequencydivision-multiple-access-(OFDMA-) based wireless mobile networks. In the single-service case, a dynamic resource 
allocation algorithm is proposed to maximize the system capacity while ensuring fairness among users. In the multiservice case, an adaptive resource allocation algorithm is proposed to support quality-of-Service-(QoS-) sensitive applications such as streaming media.

\section{Yifeng $\mathrm{He}$ \\ Ling Guan \\ Wenwu Zhu \\ Ivan Lee}



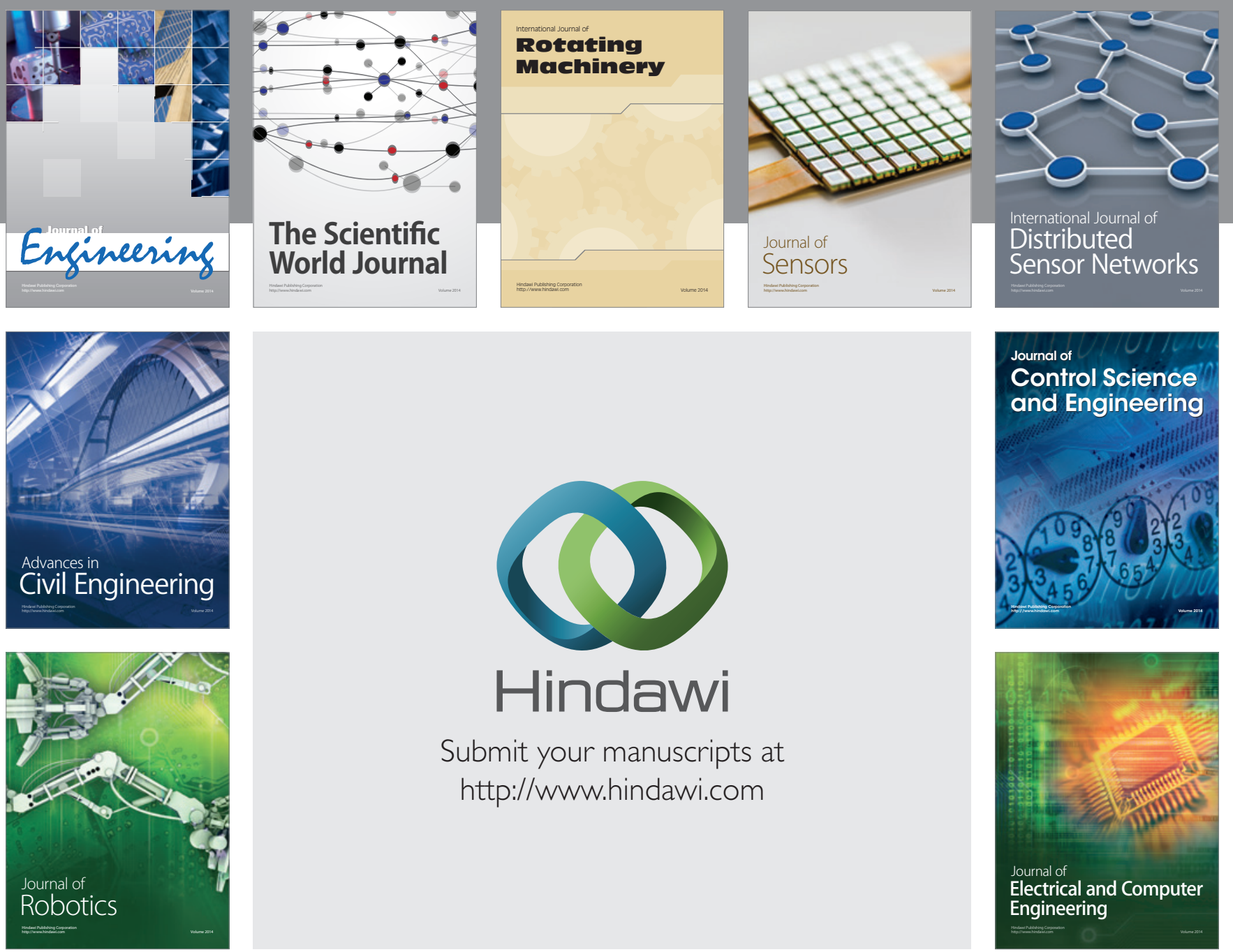

Submit your manuscripts at

http://www.hindawi.com
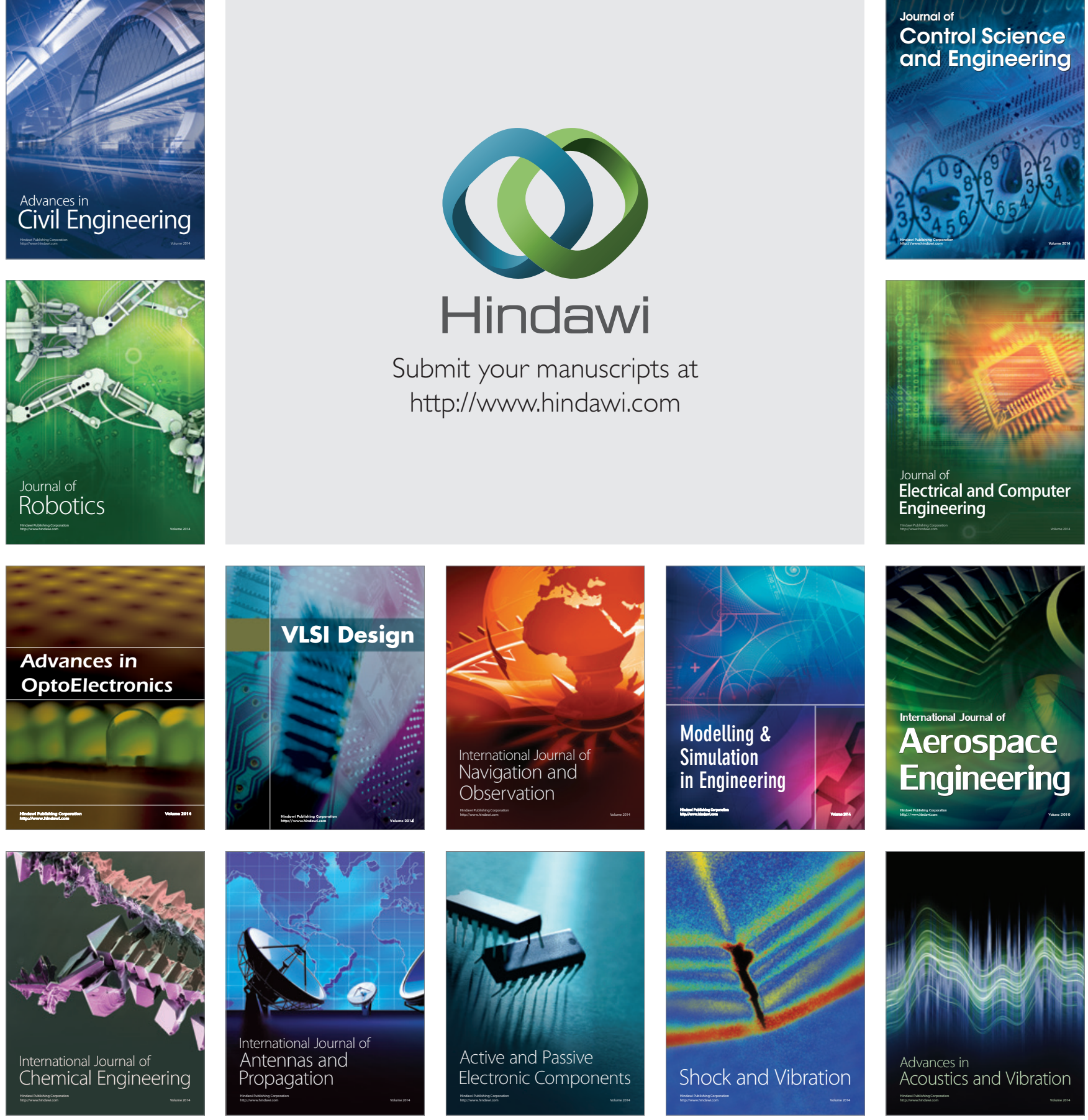